\title{
Humidity Effect on the Simulation Accuracy of Solar Vortex Engine Performance
}

\author{
Ali M. Tukkee, Hussain H. Al-Kayiem and Syed I.U. Gilani \\ Solar Thermal Advanced Research Center [STARC], Universiti Teknologi PETRONAS, 32610 Seri Iskandar, \\ Perak, Malaysia
}

\begin{abstract}
In this study, a validated computational simulation is presented to investigate the effect of humidity on the performance prediction of the solar vortex engine system. Data from an experimental model are used to validate the CFD simulation. Three humidity cases are considered: dry air, $40 \%$ and $80 \%$ humid air. An expansion process with heat addition is taking place inside the vortex generator. When the vortex field continues outside the system, a compression process with heat rejection occurs, eventually bringing the air vortex to be thermal and mechanical equilibrium with the surrounding atmosphere. The change in total energy and the heat transfer rate for both processes, inside the vortex generator and outside the vortex generator, increase with increased humidity in the working fluid. The humidity increases the energy required by the system to generate and maintain the air vortex. Compared to the dry air, the pressure drop at the center of the vortex field decreases by $(2-5 \%)$ and $(4-9 \%)$ for the $40 \%$ and $80 \%$ humid air, respectively. Reduced pressure drop decreases the stability of the air vortex when it is in contact with the atmosphere. The intensity of the air vortex is not affected by the increase in humidity.
\end{abstract}

Keywords: Humidity in solar systems, Vortex generator, Solar updraft power, Solar vortex engine, Vortex stability, Vortex intensity.

\section{INTRODUCTION}

Solar vortex power generation is a new technology that has caught the attention of researchers in the renewable energy field. It uses the same principle as the well-known solar chimney power plant, with the tall chimney structure being replaced by a vortex column. Both solar updraft systems use a solar collector to heat the air, which would then exit from the center of the collector in a continuous upward flow, either to the chimney structure or to a vortex field. A solar chimney power plant prototype was established in Manzanares / Spain in 1982, and valuable data was collected from Haaf et al. [1, 2]. Other researchers later used the data to study further and develop solar chimney technology.

On the other hand, solar vortex technology is still new, and only a few research are available in the literature. The idea of solar vortex power generation was first proposed by Michaud [3], who tried to prove the possibility and capability of such systems using the laws of thermodynamics. When heat is carried upward by convection, the work produced was equal to the convective available potential energy (CAPE). This work was calculated by Michaud using the energy equation, which he pointed out to be easier and more direct than the equation of CAPE. Michaud [4] also

*Address correspondence to this author at the Solar Thermal Advanced Research Center [STARC], Universiti Teknologi PETRONAS, 32610 Seri Iskandar, Perak, Malaysia; Tel: +605 368 7008;

E-mail: hussain_kayiem@utp.edu.my presented a thermodynamic power cycle for atmospheric upward natural convection processes. The proposed cycle was then applied for an annular tube system, which was then replaced with a vortex field. The power cycle was presented in different cases in which the air was considered dry and humid. Finally, Michaud [5] presented his design for what he called the atmospheric vortex engine. Michaud's design did not consider solar energy as the source of heat for the system. The atmospheric vortex engine uses fuel or steam to start the process that would lead to the generation of the air vortex using curved guide vanes. The air vortex would then operate a turbine to generate electricity and help in lifting heat above green house gases so that the heat can be more easily radiated to space, reducing global warming [6]. Other researchers followed and integrated the idea with solar energy as Michaud originally proposed.

A solar vortex power generation design is presented by Al-Kayiem et al. [7] named the solar vortex engine (SVE). This system uses an 8-m-diametersolar collector with a 1-m-diameter structure that houses guide vanes, called the vortex generator, at the center of the collector to create the air vortex. An experimental model is presented by Al-Kayiem et al. [7]. CFD studies are presented by different researchers [8-13] based on the system reported by Al-Kayiem et al. [7]. These simulations focused on the design and operating parameters of the system. Zhang et al. [14] introduced a device that generates a vortex field from solar energy, similar to the natural dust devils phenomena, 
that could be used as a sustainable energy source. A small-scale indoor experimental prototype validates their CFD simulation.

Another solar vortex power plant design is proposed by Ninic and Nizetic [15], named the solar power plant with a short diffuser. This design uses guide vanes located underneath the solar collector to generate the air vortex, which passes through a small structure called the short diffuser. The solar power plant with a short diffuser is studied by CFD simulation that presents the basic principle of the system and further developed the topology of the guide vanes [16, 17]. Mohiuddin and Uzgoren [18] presented a CFD study for another solar vortex power generation system to study the effect of the guide vane design parameters on the system's performance. Finally, Zuo et al. [19] presented a simulation for the idea of a solar vortex power desalination system. They only studied the system's potential for seawater desalination without actually considering the water in the simulation.

Nizetic et al. [20] conducted an experimental study on artificial vortices, using a small laboratory setup, to further understand the vortex flow field and its potential for power generation. They added the effect of humidity through injectors that introduce water to the air stream. It was found that the addition of humidity increases the energy required for maintaining the vortex field as more work is required to lift the added water. It should be noted that none of the previously mentioned CFD studies considered the effect of humidity on the vortex field, which could be a major factor in determining the energy potential of such systems.

Saadun and Sidik [21] conducted an experimental study on the climate effect on the solar chimney model with $3 \mathrm{~m}$ collector diameter and $5 \mathrm{~m}$ chimney height. They concluded that there was a significant surge in the morning humidity with an average of above $95 \%$ and the ambient temperature was approximately $35{ }^{\circ} \mathrm{C}$ due to low solar radiation. The humidity kept decreasing after sunrise due to the greenhouse effect produced in the solar collector. The study hasn't included the influence of humidity on the system performance.

Literature is lacking detailed studies on the effect of humidity on solar vortex power generation. In fact, for the best knowledge of authors, there are no related publications on the subject of humidity effect on the performance of this type of system.
Hence, in this study, a CFD procedure is developed and presented for air humidity effect on the performance of the SVE system. The computational procedure is validated by comparison with experimental measurement results. The humid climate of Malaysia is considered, and three cases for the working fluid are studied:

- $\quad$ Dry air, which is commonly adopted in the solar chimney and solar vortex simulations.

- Humid air with relative humidity $(\mathrm{RH})=40 \%$.

- Humid air with $\mathrm{RH}=80 \%$.

The article presents the related literature in the introductory section and highlights the lack of such study of humidity effect on the vortex stability and energy potential of the SVE. In the second section, the computational and experimental procedures are presented in detail. The third section presents the results highlighting the effect of humidity on the SVE performance and the type of thermodynamic processes taking place along the vortex field, inside and outside the vortex generator. The last section concludes the significant findings achieved from the research.

\section{MATERIALS AND METHODS}

\subsection{Experimental Implementation}

An experimental model was used as means of validation and acquiring input data for the CFD simulation.

\subsubsection{Model Description}

The SVE replaces the tall tower in solar chimney power plants with a small, more convenient structure called the vortex generator. The principle of the SVE is shown in Figure 1. Air moves along the solar collector and upward, exiting from the top of the collector to the vortex generator. The vortex generator consists of two concentric cylinders; the outer cylinder with 1-mdiameter and 1-m-height. The inner cylinder has $0.6-\mathrm{m}$ heightand $0.9-\mathrm{m}$, connected to the outer cylinder from the top.

At the perimeter of the inner cylinder, eight entry slots of $0.6-\mathrm{m}$-height $\times 0.1$-m-width allow the air to enter the vortex generation arena. A guide vane follows each entry slot with a $25^{\circ}$ inclination angle that converts the air stream to rotational movement generating the vortex. The air vortex then exits to the atmosphere through an upper hole. Both cylinders are 


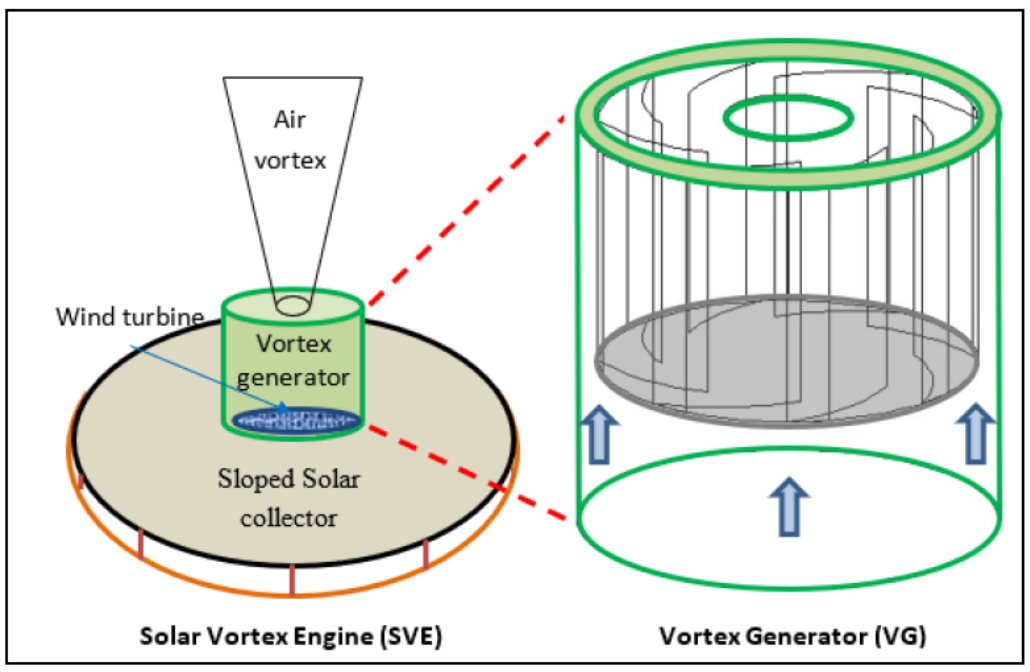

Figure 1: Outlines of the SVE.

made from Aluminum, and the interior of the inner cylinder is painted black, while the upper cover is made from a transparent Perspex sheet. The selection of the upper cover as transparent allows the penetration of solar radiation inside the vortex generator and creates a second solar energy collection mechanism. The outer cylinder is insulated to trap the thermal energy inside the system. A turbine is situated inside the lower part of the outer cylinder to capture the kinetic energy of the hot air stream and convert it to mechanical work. A generator is connected to the turbine to convert the mechanical work to electrical power.

The experimental system is shown in Figure 2, which is established at the Solar Technology Advanced Research Center (STARC) in University Teknologi PETRONAS. The system consists of a solar collector with $8 \mathrm{~m}$ diameter and inclined by $8.8^{\circ}$. The collector canopy is made from Perspex sheets that are fixed on a steel frame. The ground beneath the canopy was covered with black-painted pebbles to act as an absorption medium and a thermal energy storage system. The vortex generator is fixed at the center of the collector, and its design is as described earlier.

\subsubsection{Measurement Instruments and Data Collection}

The operational data required for the CFD simulation were collected from the experimental model measurements. Data were collected for the temperature and velocity of the air at the inlet and outlet to the vortex generator, the temperatures of the interior walls and upper cover of the vortex generator, and the ambient temperature. Thermocouples type $\mathrm{K}$ with a data logger device GRAPHTEC data logger GL820 were used for the temperature measurements. The data logger offers an accuracy of $\pm 1.7^{\circ} \mathrm{C}$ for a temperature range of $-100^{\circ} \mathrm{C}$ to $100^{\circ} \mathrm{C}$.A digital reader KIMO-AMI 300 with a hotwire probe was used for the velocity measurements. The hotwire probe can

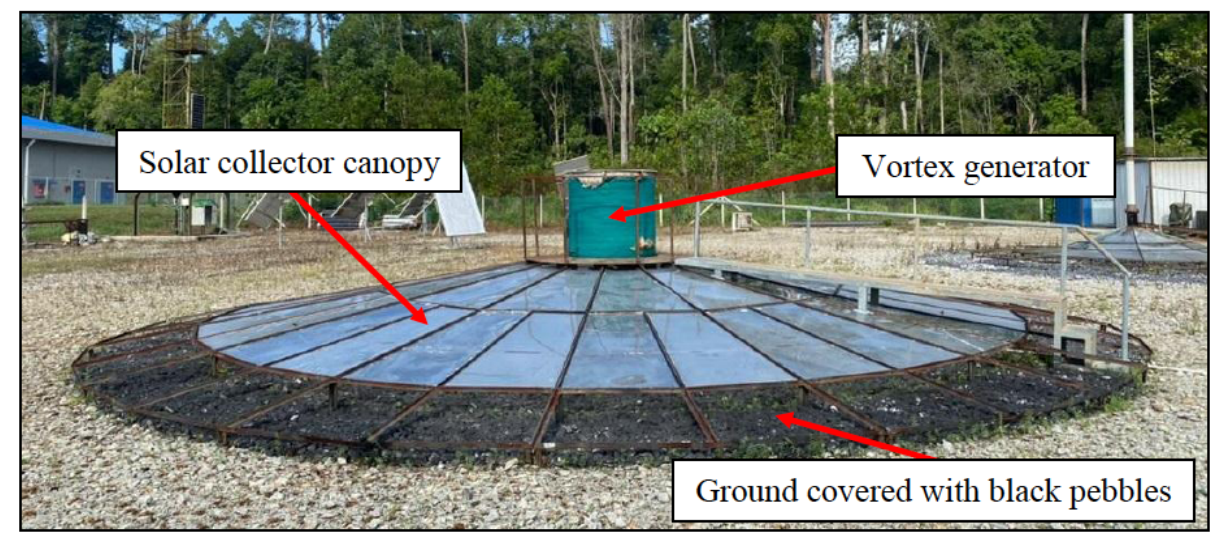

Figure 2: The solar vortex engine experimental model in the solar site of Universiti Teknologi PETRONAS. 
measure a velocity range of 0.15 to $3 \mathrm{~m} / \mathrm{s}$ with an accuracy of $\pm 3 \%$ of the reading.

The data for the temperature and velocity were collected in four days for repeatability purposes. Measurements were made every hour from 9:00 AM until 4:00 PM. The highest values of temperature and velocity were found to be reached around 2:00 PM. The measurement data were used in the numerical simulation for the boundary conditions and validation purposes.

\subsection{Computational Procedure}

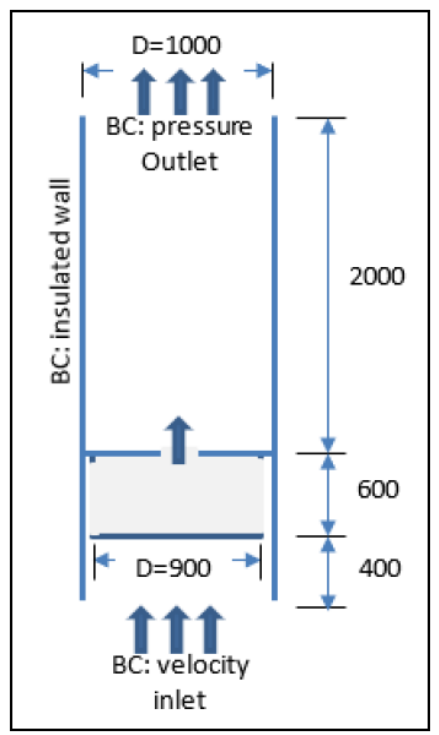

Figure 3: Computational model.

The vortex generator, representing a fixed swirling air generation unit in the SVE, has been considered the main thermo-fluid changer in a CFD simulation. The computational simulation was achieved by solving the governing equations using ANSYS Fluent 2020 R1 software. The computational model of the investigated SVE is shown in Figure 3.

\subsubsection{Computational Domain and Meshing Method}

The computational domain was created with AutoCAD software, and the mesh was generated using ANSYS ICEM. The system geometry was divided into four parts: two for the outer cylinder, one for the inner cylinder and one for an exterior vortex domain of 2-mheight. The exterior domain allows the study of the generated air vortex outside the system. Structured hexahedral meshing was used with few prism cells in the inner cylinder, and the multizone meshing method was adopted. The four parts of the geometry are connected by contact regions defined as matching interfaces in the Fluent solver. A single inflation layer was added to ensure the value of $\mathrm{y}+$ be within the required range for the turbulence model, while the Menter-Lechner insensitive $y+$ wall treatment was used. Figure 4 shows the geometry representing the computational domain and a section of the mesh of the vortex generator.

The mesh independence study was achieved by taking different cell sizes, which changes the overall number of cells. The mass flow rate at $0.1 \mathrm{~m}$ above the outer hole was compared at each size. When the difference became insignificant, the chosen cell size was identified. It was found that when the number of cells changes from 439020 to 511646 , the change in mass flow rate was $0.08 \%$, so the mesh size with 439020 cells was used in the study.

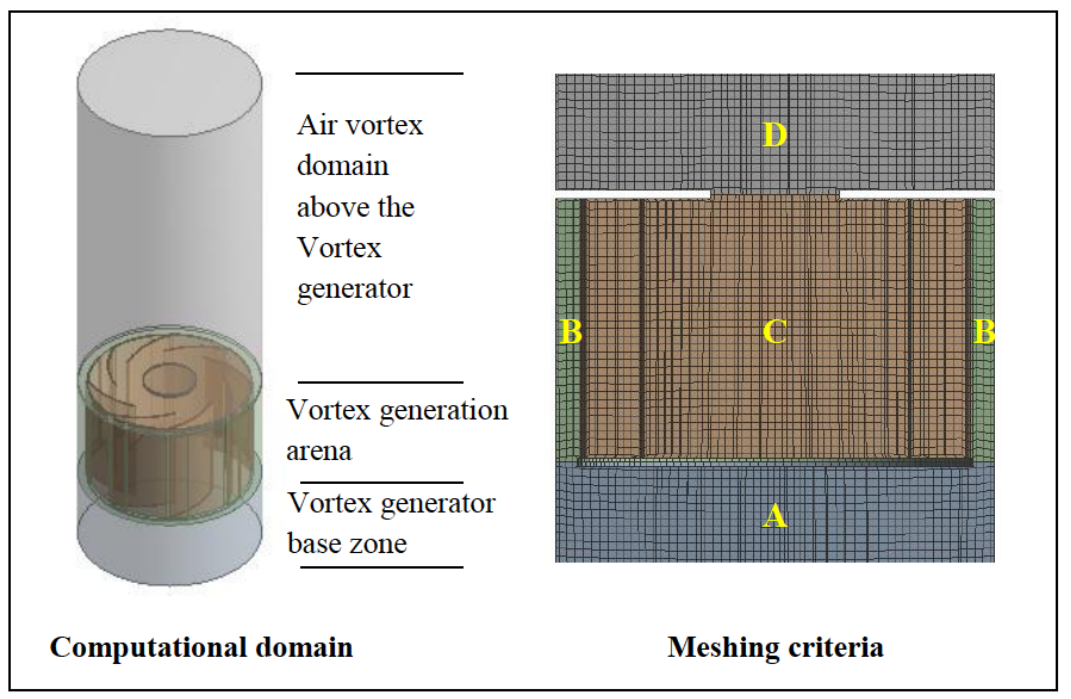

Figure 4: Computational domain and meshing zones of the system, each consist of four parts: (A). the lower part of the outer cylinder, (B). annulus area between the inner and outer cylinders, (C). inner cylinder and (D). vortex domain. 
Continuity equation

$\frac{1}{r} \frac{\partial\left(\rho V_{r} r\right)}{\partial r}+\frac{1}{r} \frac{\partial\left(\rho V_{\theta}\right)}{\partial \theta}+\frac{\partial\left(\rho V_{Z}\right)}{\partial z}=0$

Momentum equation, in $r, \theta, z$

$$
\begin{aligned}
& \frac{1}{r} \frac{\partial\left(\rho V_{r}^{2} r\right)}{\partial r}+\frac{1}{r} \frac{\partial\left(\rho V_{r} V_{\theta}\right)}{\partial \theta}+\frac{\partial\left(\rho V_{r} V_{z}\right)}{\partial z}=-\frac{\partial p}{\partial r}+\frac{1}{r} \frac{\partial\left(\tau_{r r} r\right)}{\partial r}+\frac{1}{r} \frac{\partial\left(\tau_{r \theta}\right)}{\partial \theta}+\frac{\partial\left(\tau_{r z}\right)}{\partial z}+\frac{\tau_{\theta \theta}}{r}+\frac{\rho V_{\theta}^{2}}{r}+\rho g_{r} \\
& \frac{1}{r} \frac{\partial\left(\rho V_{\theta} V_{r} r\right)}{\partial r}+\frac{1}{r} \frac{\partial\left(\rho V_{\theta}^{2}\right)}{\partial \theta}+\frac{\partial\left(\rho V_{\theta} V_{z}\right)}{\partial z}+\frac{\rho V_{r} V_{\theta}}{r}=-\frac{\partial p}{\partial \theta}+\frac{1}{r^{2}} \frac{\partial\left(\tau_{\theta r} r^{2}\right)}{\partial r}+\frac{1}{r} \frac{\partial\left(\tau_{\theta \theta}\right)}{\partial \theta}+\frac{\partial\left(\tau_{\theta z}\right)}{\partial z}+\rho g_{\theta} \\
& \frac{1}{r} \frac{\partial\left(\rho V_{z} V_{r} r\right)}{\partial r}+\frac{1}{r} \frac{\partial\left(\rho V_{z} V_{\theta}\right)}{\partial \theta}+\frac{\partial\left(\rho V_{z}^{2}\right)}{\partial z}=-\frac{\partial p}{\partial z}+\frac{1}{r} \frac{\partial\left(\tau_{z r} r\right)}{\partial r}+\frac{1}{r} \frac{\partial\left(\tau_{z \theta}\right)}{\partial \theta}+\frac{\partial\left(\tau_{z z}\right)}{\partial z}+\rho g_{z} \text { (2-C) }
\end{aligned}
$$

Energy equation

$$
\begin{aligned}
& \frac{1}{r} \frac{\partial\left(V_{r} E_{t} r\right)}{\partial r}+\frac{1}{r} \frac{\partial\left(V_{\theta} E_{t}\right)}{\partial \theta}+\frac{\partial\left(V_{z} E_{t}\right)}{\partial z}=\rho \dot{q}-\frac{1}{r} \frac{\partial\left(q_{r} r\right)}{\partial r}-\frac{1}{r^{2}} \frac{\partial\left(q_{\theta}\right)}{\partial \theta}-\frac{\partial\left(q_{z}\right)}{\partial z}-\frac{1}{r} \frac{\partial\left(V_{r} p r\right)}{\partial r}-\frac{1}{r} \frac{\partial\left(V_{\theta} p\right)}{\partial \theta}- \\
& \frac{\partial\left(V_{z} p\right)}{\partial z}+\frac{1}{r} \frac{\partial\left(V_{r} \tau_{r r} r\right)}{\partial r}+\frac{1}{r} \frac{\partial\left(V_{r} \tau_{r \theta}\right)}{\partial \theta}+\frac{\partial\left(V_{r} \tau_{r z}\right)}{\partial z}+\frac{V_{r} \tau_{\theta \theta}}{r}+\frac{1}{r^{2}} \frac{\partial\left(V_{\theta} \tau_{\theta r} r^{2}\right)}{\partial r}+\frac{1}{r} \frac{\partial\left(V_{\theta} \tau_{\theta \theta}\right)}{\partial \theta}+\frac{\partial\left(V_{\theta} \tau_{\theta z}\right)}{\partial z}+ \\
& \frac{1}{r} \frac{\partial\left(V_{z} \tau_{z r} r\right)}{\partial r}+\frac{1}{r} \frac{\partial\left(V_{z} \tau_{z \theta}\right)}{\partial \theta}+\frac{\partial\left(V_{z} \tau_{z z}\right)}{\partial z}+\rho\left(V_{r} g_{r}+V_{\theta} g_{\theta}+V_{z} g_{z}\right)
\end{aligned}
$$

\subsubsection{Governing Equations and Solution Methods}

The governing equations solved in the simulation are the conservation of mass, momentum and energy. According to the simulation assumptions, steady and $3 \mathrm{D}$, the governing equations are as presented in the set of equations ( 1 ) to:

Where,

$$
\begin{aligned}
& E_{t}=\rho\left(e+\frac{V^{2}}{2}\right) \\
& \tau_{r r}=2 \mu \frac{\partial V_{r}}{\partial r}-\frac{2}{3} \mu\left(\frac{\partial V_{r}}{\partial r}+\frac{\partial V_{z}}{\partial z}+\frac{V_{r}}{r}\right) \\
& \tau_{\theta \theta}=-2 \mu \frac{V_{r}}{r}-\frac{2}{3} \mu\left(\frac{\partial V_{r}}{\partial r}+\frac{\partial V_{z}}{\partial z}+\frac{V_{r}}{r}\right) \\
& \tau_{z z}=2 \mu \frac{\partial V_{z}}{\partial z}-\frac{2}{3} \mu\left(\frac{\partial V_{r}}{\partial r}+\frac{\partial V_{z}}{\partial z}+\frac{V_{r}}{r}\right) \\
& \tau_{r z}=\tau_{z r}=\mu\left(\frac{\partial V_{z}}{\partial r}+\frac{\partial V_{r}}{\partial z}\right) \\
& \tau_{r \theta}=\tau_{\theta r}=\mu r\left(\frac{\partial\left(V_{\theta} / r\right)}{\partial r}\right) \\
& \tau_{\theta z}=\tau_{z \theta}=\mu\left(\frac{\partial V_{\theta}}{\partial z}\right) \\
& q_{r}=-k \frac{\partial T}{\partial r} \\
& q_{z}=-k \frac{\partial T}{\partial z}
\end{aligned}
$$

The realizable $k-\varepsilon$ turbulence model was used, which uses two transport equations for the turbulence kinetic energy, $k$ and its rate if dissipation, $\varepsilon$ :

$$
\frac{\partial}{\partial x_{j}}\left(\rho k u_{j}\right)=\frac{\partial}{\partial x_{j}}\left[\left(\mu+\frac{\mu_{t}}{\sigma_{k}}\right) \frac{\partial k}{\partial x_{j}}\right]+G_{k}+G_{b}-\rho \varepsilon-Y_{M}+S_{k}
$$

$\frac{\partial}{\partial x_{j}}\left(\rho \varepsilon u_{j}\right)=\frac{\partial}{\partial x_{j}}\left[\left(\mu+\frac{\mu_{t}}{\sigma_{\varepsilon}}\right) \frac{\partial \varepsilon}{\partial x_{j}}\right]+\rho C_{1} S \varepsilon-\rho C_{2} \frac{\varepsilon^{2}}{k+\sqrt{v \varepsilon}} C_{1 \varepsilon} \frac{\varepsilon}{k} C_{3 \varepsilon} G_{b}+S_{\varepsilon}$

The turbulent viscosity, $\mu_{t}$ is computed by combining $k$ and $\varepsilon$ as follows:

$\mu_{t}=\rho C_{\mu} \frac{k^{2}}{\varepsilon}$

The system is assumed to be 3 dimensional, incompressible and steady-state. The variation of density of the working fluid with temperature is calculated using the incompressible ideal gas law. The incompressible ideal gas law allows the inclusion of species transport equations. The species transport equations were used to add the effect of humidity on the working fluid. Three cases for the working fluid are considered: dry air and humid air with $\mathrm{RH}$ values of $40 \%$ and $80 \%$. The average annual $\mathrm{RH}$ in Malaysia is $80 \%$ [22], while the $40 \%$ case is considered for comparison reasons.

The pressure-based segregated (SIMPLE) algorithm was used with the third-order MUSCL spatial 
discretization. The convergence residuals were set to $10^{-4}$ for all equations except the energy equation, set to $10^{-6}$. Further convergence was monitored by creating monitors for the air's physical properties and checking the system's mass and energy imbalances. Convergence for the residuals was achieved at 1904 , 2219 and 2276 iterations for the dry air, 40\% humid air and $80 \%$ humid air, respectively. The calculations were allowed to continue until all the important physical properties, velocity, pressure, temperature and density, reached a steady state, which was at 3000 iterations in all three cases.

\subsubsection{Boundary Conditions}

The boundary conditions are shown in Table 1, and their values were obtained from the experimental model. All the walls of the system were given zero thickness, so no conduction heat transfer was considered. The exterior vortex domain wall was given a constant temperature boundary condition with a value of the ambient temperature to play the role of a heat sink representing the surrounding environment.

Table 1: Boundary Conditions Adopted in the CFD Simulation

\begin{tabular}{|c|c|}
\hline Part & Boundary Condition \\
\hline \hline Inlet & Velocity inlet \\
\hline Outer cylinder walls & Insulated \\
\hline Inner cylinder walls & Constant temperature \\
\hline Inner cylinder lower cover & Constant temperature \\
\hline Upper cover & Constant temperature \\
\hline Guide vane walls & Constant temperature \\
\hline Exterior vortex domain wall & Constant temperature \\
\hline Exterior vortex domain outlet & Outflow \\
\hline
\end{tabular}

\section{RESULTS AND DISCUSSIONS}

The results presented in this section explore the effect of humidity on the energy potential of the SVE and the stability and intensity of the generated air vortex. The results of the numerical simulation were validated by comparison with the experimental data. The percentage of increase in velocity magnitude between the inlet from the entry slots and the outlet from the upper hole were compared. Increases in velocity of $88.7 \%$ and $86.7 \%$ were obtained from the simulation results and experimental data, respectively.

\subsection{Types of Processes Along the Vortex Field}

In Figure 5, three states of the air along the vortex generator, detailed in Table 2. Two thermodynamic processes will be considered. The first process is from state 1 to state 2, named process (1-2) and will occur entirely inside the vortex generator. The second process will take place outside the vortex generator along the exterior domain from state 2 to state 3 , and it will be named process (2-3). The air flows upward inside the vortex generator while being heated, and its movement is converted to a swirly motion by the guide vanes. The air vortex then continues its upward movement outside the system to the end of the exterior vortex domain while losing heat to the surroundings.

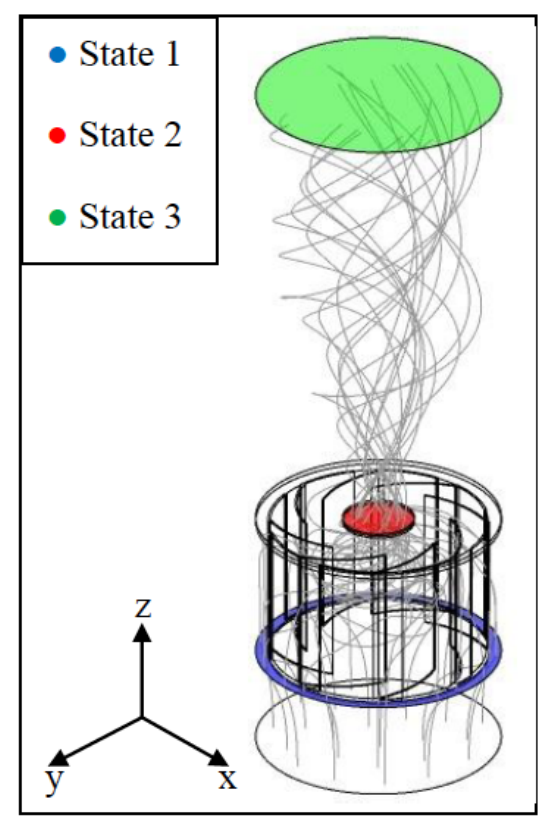

Figure 5: Air states along with the vortex generator.

Figure 6 presents the pressure contours and the variation in pressure along the center of the air vortex inside and outside the system, starting from the bottom, $z=0.0 \mathrm{~m}$ of the inner cylinder to the end of the vortex domain, $z=3.0 \mathrm{~m}$. Only the case of dry air is considered in Figure $\mathbf{5}$ as the pressure variation due to the humidity factor is discussed in section 3.3. The figure shows that the pressure decreases as air moves upward, and the vortex is generated inside the inner cylinder. The pressure then starts increasing along with the vortex domain outside the system. This means that an expansion process with heat addition occurs inside the vortex generator, while a compression process with heat rejection occurs along with the exterior vortex domain. A severe pressure drop can be seen at the exit 
Table 2: States of the Air Along the Vortex Field

\begin{tabular}{|c|c|c|}
\hline State & Description & Location with Respect to the Vertical Axis (z-axis) \\
\hline \hline State 1 & $\begin{array}{r}\text { Airflow conditions at the entrance to the annulus } \\
\text { region between the outer and inner cylinders. }\end{array}$ & $\mathrm{z}=0.0 \mathrm{~m}$ \\
\hline State 2 & $\begin{array}{r}\text { Air vortex conditions at the exit of the vortex } \\
\text { generator from the upper plate hole. }\end{array}$ & $\mathrm{z}=1.0 \mathrm{~m}$ \\
\hline State 3 & Air vortex conditions at the top of the exterior \\
vortex domain.
\end{tabular}

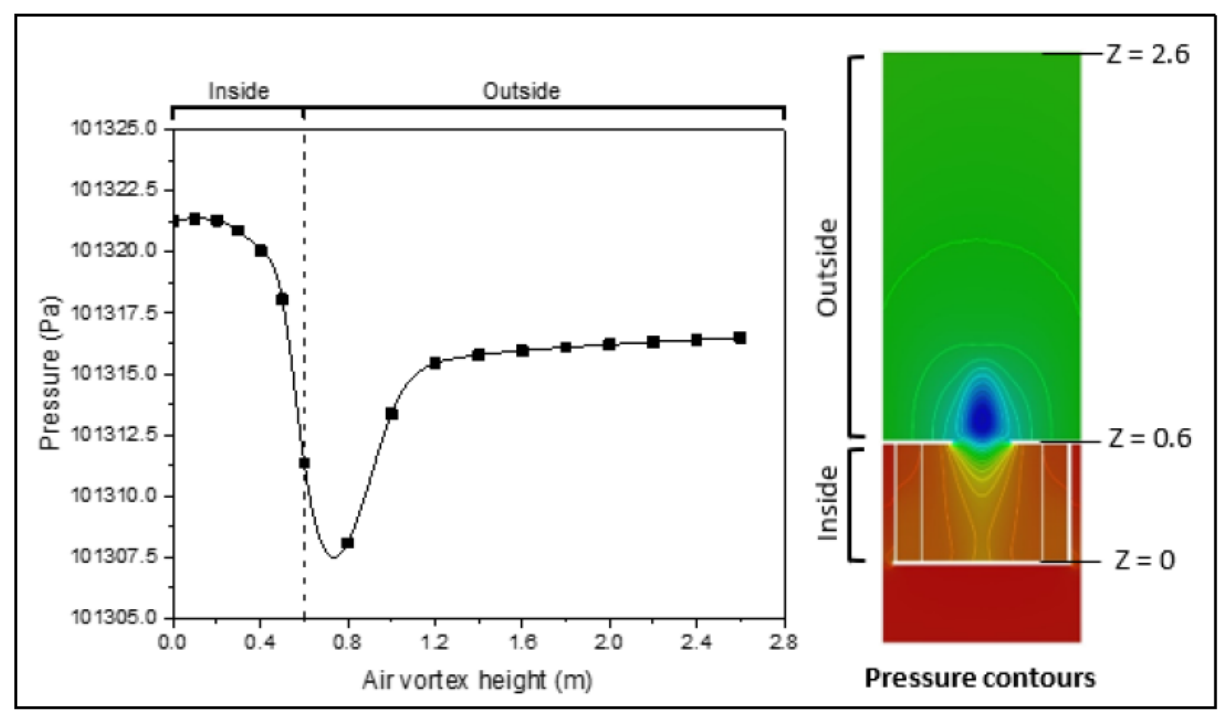

Figure 6: Variation of air pressure along the upward of the vortex field inside and outside the vortex generator. ( $\mathrm{z}$ in meters).

from the vortex generator, which is attributed to the nozzle effect of the upper hole. Other than that, the decrease and increase in pressure inside and outside the vortex generator, respectively, is uniform.

\subsection{Thermal Analysis}

\subsubsection{Effect of Humidity on Heat Gain Prediction}

The path of each process from starting state to the other state is determined by the heat and work transfer values during each process. In both processes, there is no shaft work to be considered. The heat transfer rate and the processes with different humidity values are shown in Tables $\mathbf{3}$ and $\mathbf{4}$. The positive sign means heat is added to the system, while the negative sign means heat rejection. The heat transfer for both processes seems to increase with the increase in air humidity. This heat transfer increment means that humid air requires more heat than dry air to reach the same temperature. At the same time, it loses more heat when in contact with the atmosphere.
Table 3: Heat Transfer Rate Along the Process (1-2)

\begin{tabular}{|c|c|c|c|}
\hline Variable & Dry air & $\mathbf{R H}=\mathbf{4 0} \%$ & $\mathbf{R H}=\mathbf{8 0} \%$ \\
\hline \hline (Watt) & 888.1 & 891.1 & 894.1 \\
\hline$\%$ of change & - & $0.3 \%$ & $0.67 \%$ \\
\hline
\end{tabular}

Table 4: Heat Transfer Rate Along the Process (2-3)

\begin{tabular}{|c|c|c|c|}
\hline Variable & Dry air & $\mathbf{R H}=\mathbf{4 0} \%$ & $\mathbf{R H}=\mathbf{8 0} \%$ \\
\hline \hline (Watt) & -693.9 & -696.3 & -698.6 \\
\hline$\%$ of change & - & $0.43 \%$ & $0.7 \%$ \\
\hline
\end{tabular}

Based on the simulation prediction, the areaweighted average temperature of the air at the three states for the different humidity cases is shown in Figure 7. Temperature values denote that almost the same temperature is reached for all humidity cases despite the difference in heat transfer rates. The driving force for solar vortex systems is the buoyancy force of the air, which is directly affected by the temperature 
value. This means that the system requires more thermal energy to raise humid air than dry air.

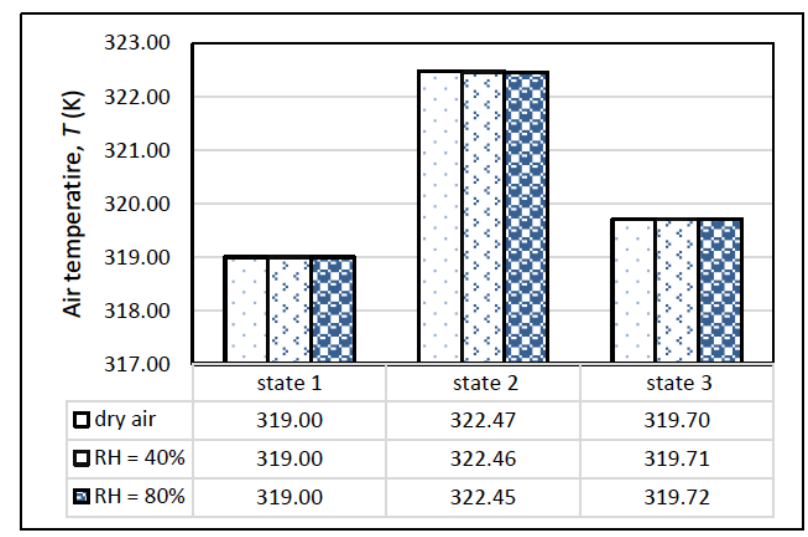

Figure 7: The predicted area-weighted average temperature of the air at the three states for the different humidity cases.

\subsubsection{Effect of Humidity on Enthalpy Prediction}

The predicted variation of the air enthalpy inside and outside the vortex generator for the three humidity cases is shown in Figure 8. In all three cases, the enthalpy behavior is the same as it decreases with the upward movement of the air. The enthalpy reduction is due to the decrease in temperature as the air inside the vortex generator moves further from the interior walls and loses heat to the surrounding atmosphere. The enthalpy seems to increase with humidity and as more water vapor is added to the air. The higher enthalpy of the humid air means that there is more energy to be consumed for the generation and maintenance of the vortex field as compared to the dry air case. An increase of about $2 \%$ in the enthalpy value is found between the dry and $40 \%$ humid air cases, whereas about $4 \%$ increase can be observed between the dry and $80 \%$ humid air cases.

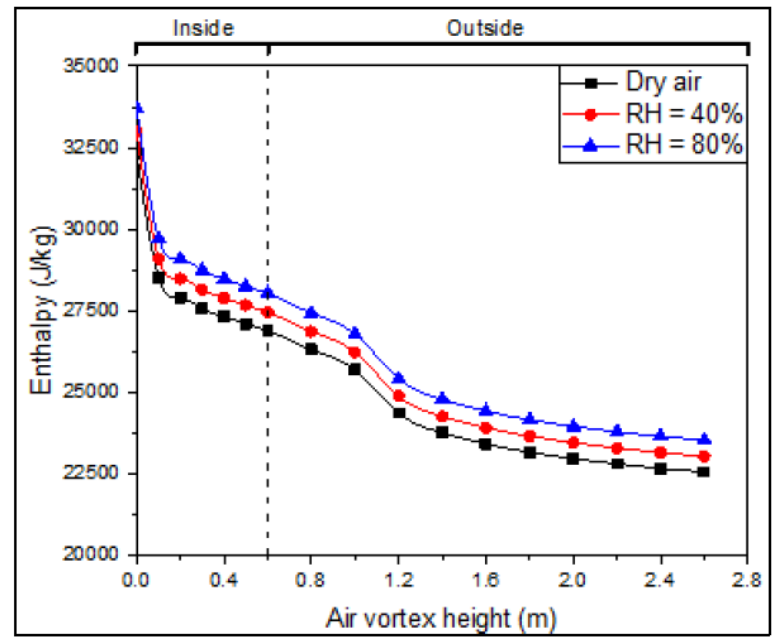

Figure 8: Variation of air enthalpy along the vortex field inside and outside the vortex generator.
Figure 9 presents the simulation predicted enthalpy values at each of the three states. Figure 10 presents the mean airflow velocity at states 1 , state 2 and state3. The specific enthalpy and velocity at each state are taken as the area-weighted average values. The difference in the elevation in the flow direction, $\Delta z$ is taken as $0.6 \mathrm{~m}$ and $2 \mathrm{~m}$ for the processes (1-2) and (2$3)$, respectively.

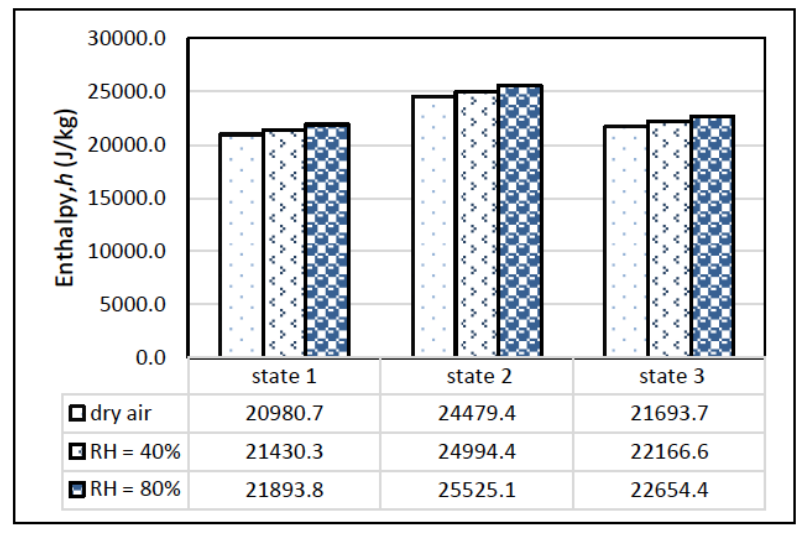

Figure 9: The area-weighted predicted values of the enthalpy at each state.

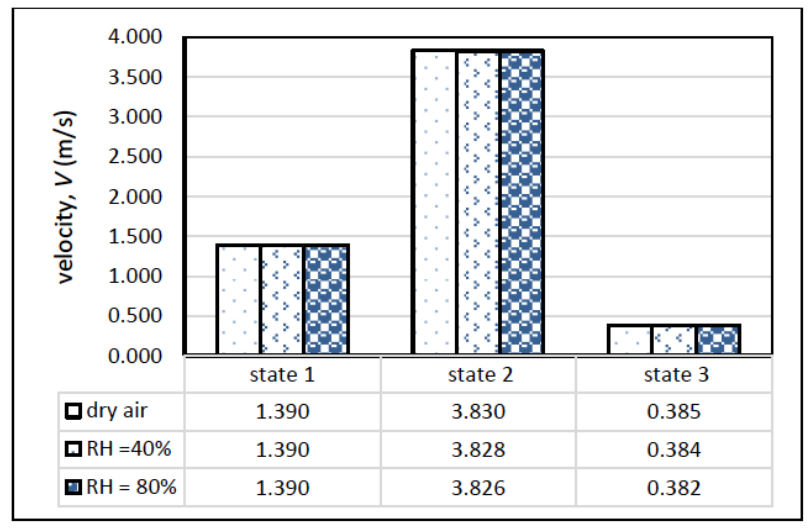

Figure 10: The area-weighted predicted values of the airflow velocity at each state.

\subsubsection{Effect of Humidity on Total Energy Prediction}

When the movement of air along the vortex field is considered as a thermodynamic process, the change in total energy, $\Delta E$ is representing the sum of changes in the thermal energy, kinetic energy and potential energy, as in equation 7 :

$\Delta E=\Delta h+\frac{1}{2} \Delta V^{2}+g \Delta z$

Figure 11 shows that the change in total energy, $\Delta E_{1-2}$ for the process 1-2, increases as humidity is added to the air. It could be noticed that for the process 2-3 that, also there is an increase in the total energy 
change $\Delta E_{2-3}$ as more humidity is added to the air. However, the positive sign of $\Delta E$ during process 1-2 means that energy is added to the air inside the vortex generator, while the negative sign means that energy is being lost to the surrounding along the process 2-3.

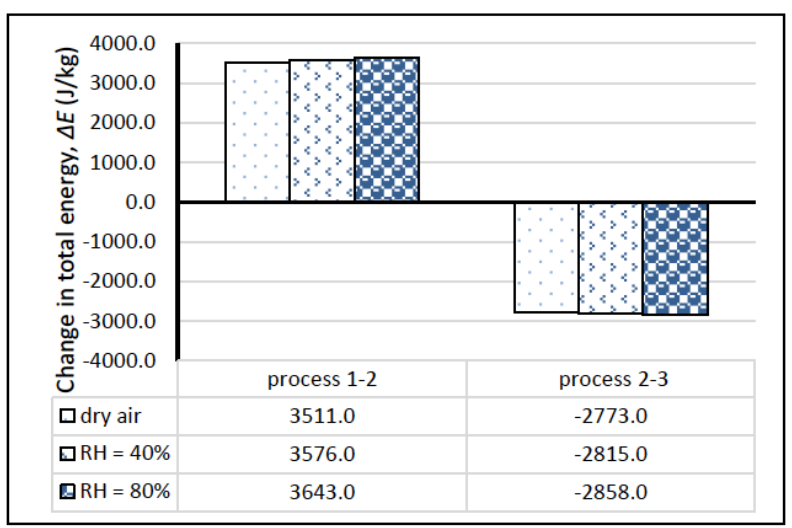

Figure 11: The predicted values of the total energy change in the airflow during process 1-2 and process 2-3, for three different humidity cases.

The energy required to raise the air parcels and generate and maintain the air vortex increases as more humidity is added. Similar findings are reported by Nizetic et al. [20] in their experimental study. In solar vortex systems, the energy gained at the solar collector is divided between operating the turbine and generating the air vortex. The power required for the turbine can be determined by the type and size of the turbine. Suppose the energy required for the generation of the air vortex is obtained from a CFD simulation. In that case, we can determine the size and design parameters of the solar collector from the overall power requirements. This is very important as the collector takes a large amount of the overall cost of such a plant construction. So, the humidity effect is important to accurately determine the thermal energy requirements and sequentially the size and cost of the power plant.

\subsection{Stability and Intensity Analysis of the Air Vortex}

Two parameters could be used to evaluate the stability and intensity of the vortex field; the pressure drop at the center of the vortex and the vorticity magnitude at the outer boundary of the vortex.

\subsubsection{Effect of Humidity on Pressure Prediction}

Figure 12 presents contour distributions of these parameters at $1 \mathrm{~m}$ height above the outlet hole. Both the low pressure at the center of the air vortex and the rotational motion at its outer boundary help in maintaining the vortex column against the atmospheric compression exerted on it. So, higher values of each of these parameters mean better stability and higher intensity.

Figure 13 shows the pressure drop values for the three humidity cases along the vortex height, starting from the outlet hole at $0.6 \mathrm{~m}$ height in the z-axis direction. It can be seen that the pressure drop is lower for humid air and decreases further as more water vapor is added to the air. A decrease of about $2-5 \%$ in the pressure drop occurs when the humidity is increased to $40 \%$ compared to dry air, while a decrease of about $4-9 \%$ occurs with $80 \%$ humidity

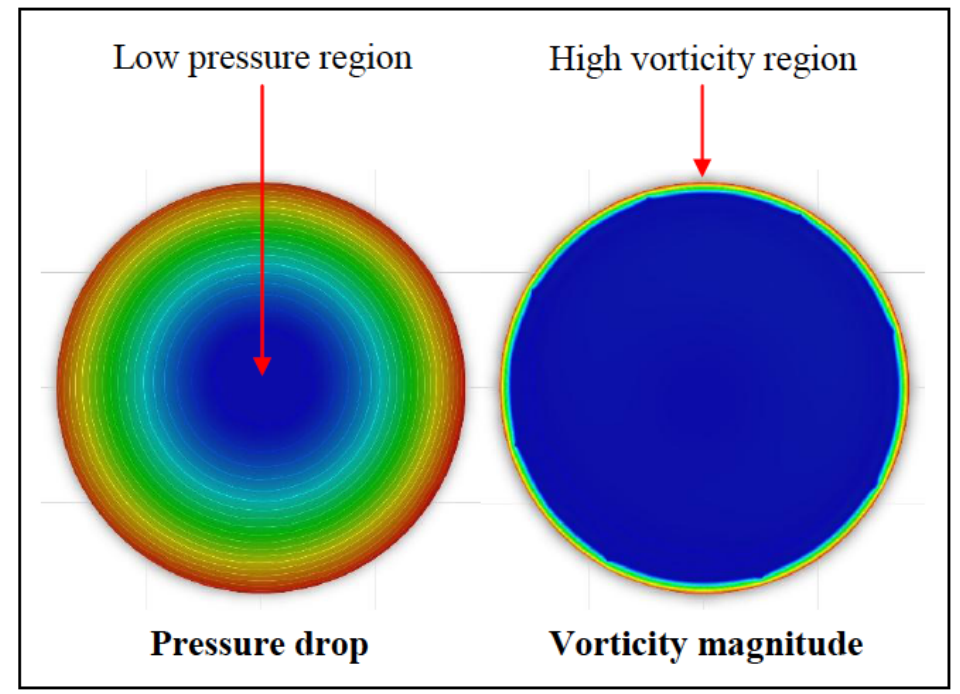

Figure 12: Distribution of vorticity magnitude and pressure drop along the diameter of the vortex field at $1 \mathrm{~m}$ above the outlet hole. 
compared to dry air. This will cause the vortex field to be less stable in higher humidity regions as compared to dry regions.

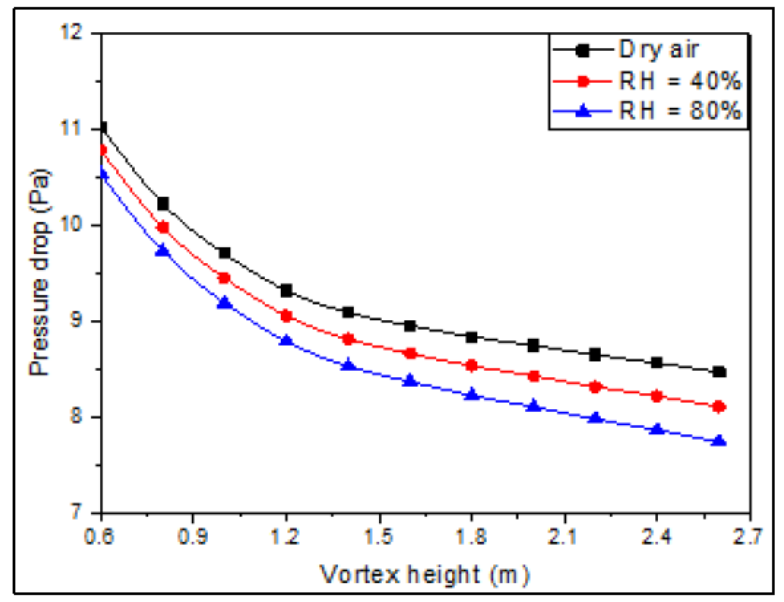

Figure 13: Pressure drop along with the vortex height outside the vortex generator.

\subsubsection{Effect of Humidity on Vorticity Prediction}

The vorticity magnitude of the air along the vortex field is shown in Figure 14. The vorticity is a measure of the vortex intensity, as the intensity is equal to the vorticity magnitude multiplied by the cross-sectional area. Higher vorticity can be seen for the humid air below $1 \mathrm{~m}$ height, after which the dry air produces higher velocity values. The difference in vorticity magnitude between the three humidity cases seems to be very small in values. So, the effect of humidity on the vortex intensity is insignificant when studying solar vortex power generation systems.

\section{CONCLUSIONS}

This study presented a CFD simulation for a solar vortex generator system as part of the SVE. Three cases for the working fluid were considered: dry air and humid air with $\mathrm{RH}=40 \%$ and $\mathrm{RH}=80 \%$. The results showed that humidity could affect the performance prediction of the SVE system, and the following was concluded:

- $\quad$ An expansion process with heat addition takes place inside the vortex generator, while a compression with heat rejection occurs when the air vortex exits the system to the atmosphere.

- The system requires more thermal energy to generate and raise the air vortex through the atmosphere when humidity is considered.

- $\quad$ The humidity of the air plays an important role in the energy required to generate and maintain the vortex field, which affects the energy potential of the system.

- $\quad$ The stability of the air vortex, evaluated through the pressure drop, decreases as more water vapor is added to the air.

- The intensity of the vortex field, evaluated by the vorticity magnitude, is unaffected by the air humidity.

It is suggested that future CFD studies on solar vortex power generation systems consider the

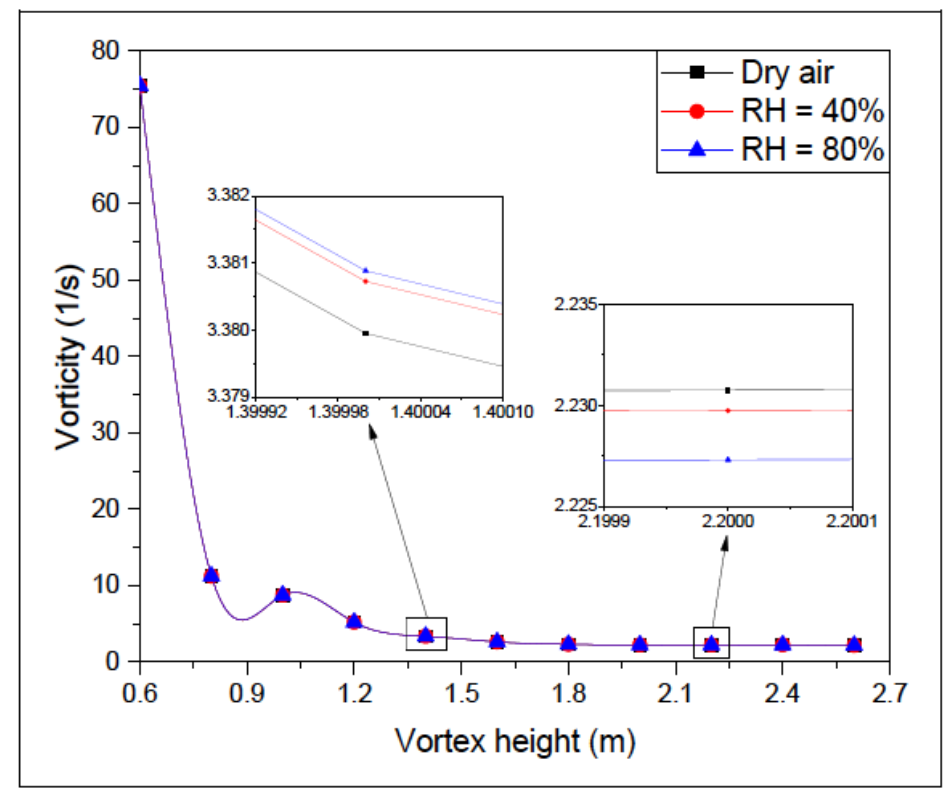

Figure 14: Vorticity magnitude along the vortex height outside the vortex generator. 
existence of water vapor in the air to estimate the energy potential of such systems accurately.

\section{ACKNOWLEDGMENTS}

The authors would like to acknowledge PETRONAS for the financial assistance under the research grant by YUTP - FRG, CS: 015LC0 - 026 to carry out the research. The first author highly appreciates UTP support to carry out his PhD study under the Graduate Assistantship (GA) scheme.

\section{NOMENCLATURE}

\section{Symbols}

$C_{1} \quad$ Variable of the dissipation rate equation

$C_{2}, C_{1 \varepsilon}$ Constants

$C_{3 \varepsilon} \quad$ Degree to which the dissipation rate is affected by the buoyancy

$C_{\mu} \quad$ Variable of eddy viscosity formula

E Energy, J

$\vec{F} \quad$ External body forces

g Gravitational acceleration, $\mathrm{m} / \mathrm{s}^{2}$

$G_{k} \quad$ Generation of turbulence kinetic energy due to the mean velocity gradients

$G_{b} \quad$ Generation of turbulence kinetic energy due to buoyancy

$h \quad$ Enthalpy, J

$J \quad$ Species

$K \quad$ Turbulence kinetic energy, $\mathrm{m}^{2} / \mathrm{s}^{2}$

$P \quad$ Pressure, $\mathrm{Pa}$

$\dot{Q} \quad$ Heat transfer rate, (Watt)

RH Relative humidity

$S \quad$ Modulus of the mean rateofstrain tensor

$S_{m} \quad$ Source term for the continuity equation

$S_{h} \quad$ Source term for the energy equation

$S_{k}, S_{\varepsilon} \quad$ Source terms for turbulence equations
$T \quad$ Temperature, $\mathrm{K}$

$V \quad$ Velocity, $\mathrm{m} / \mathrm{s}$

$\vec{v} \quad$ Velocity vector, $\mathrm{m} / \mathrm{s}$

$Y_{M} \quad$ Contribution of the fluctuating dilatation incompressible turbulence to theoverall dissipation rate

$z \quad$ Elevation, $\mathrm{m}$

\section{Greek Symbols}

$\varepsilon \quad$ Dissipation rate of kinetic energy, $\mathrm{m}^{2} / \mathrm{s}^{3}$

$\mu \quad$ Viscosity, N.s $/ \mathrm{m}^{2}$

$\mu_{t} \quad$ Turbulent viscosity, $\mathrm{m}^{2} / \mathrm{s}$

$v \quad$ Kinematic viscosity, $\mathrm{m}^{2} / \mathrm{s}$

$\rho \quad$ Density, $\mathrm{kg} / \mathrm{m}^{3}$

$\sigma_{k}, \sigma_{\varepsilon} \quad$ Turbulent Prandtl numbers for $k$ and $\varepsilon$

$\overline{\bar{\tau}} \quad$ Stress tensor, $\mathrm{N} / \mathrm{m}^{2}$

\section{Abbreviations}

$C A P E$ Convective available potential energy

CFD Computational fluid dynamics

SVE Solar vortex engine

\section{REFERENCES}

[1] W. Haaf, K. Friedrich, G. Mayr, J. Schlaich, Solar chimneys part I: principle and construction of the pilot plant in Manzanares, International Journal of Solar Energy 1983; 2(1): 3-20. https://doi.org/10.1080/01425918308909911

[2] W. Haaf, Solar chimneys: part ii: preliminary test results from the Manzanares pilot plant, International Journal of Sustainable Energy 1984; 2(2): 141-161. https://doi.org/10.1080/01425918408909921

[3] LM. Michaud, Proposal for the use of a controlled tornadolike vortex to capture the mechanical energy produced in the atmosphere from solar energy, Bulletin of the American Meteorological Society 1975; 530-534.

[4] LM. Michaud, Thermodynamic cycle of the atmospheric upward heat convection process, Meteorology and Atmospheric Physics 2000; 72(1): 29-46. https://doi.org/10.1007/s007030050003

[5] LM. Michaud, Atmospheric vortex engine, Google Patents, 2006.

[6] LM. Michaud, The atmospheric vortex engine, 2009 IEEE Toronto International Conference Science and Technology for Humanity (TIC-STH), IEEE, 2009; pp. 971-975. https://doi.org/10.1109/TIC-STH.2009.5444355

[7] HH. Al-Kayiem, and Mustafa, AT. Solar Vortex Engine, 
Malaysia Patent; PI2015702890, 2015.

[8] HH. Al-Kayiem, AT. Mustafa, SI. Gilani, Solar vortex engine: Experimental modelling and evaluation, Renewable Energy 2018; 121: 389-399. https://doi.org/10.1016/j.renene.2018.01.051

[9] A. Ismaeel, H. Al-Kayiem, A. Baheta, M. Aurybi, Numerical analysis on the influence of inflow guide vanes in A solar vortex power generator, WIT Transactions on Ecology and the Environment 2017; 224: 553-563. https://doi.org/10.2495/ESUS170511

[10] P. Das, V. Chandramohan, Estimation of flow parameters and power potential of solar vortex engine (SVE) by varying its geometrical configurations: A numerical study, Energy Conversion and Management 2020; 223: 113272. https://doi.org/10.1016/j.enconman.2020.113272

[11] P. Das, Performance evaluation of solar vortex engine and optimization of number of air entry slots and turbine location, Energy Sources, Part A: Recovery, Utilization, and Environmental Effects 2020; 1-17. https://doi.org/10.1080/15567036.2020.1845879

[12] AA. Ismaeel, HH. Al-Kayiem, AT. Baheta, MA. Aurybi, CFD modeling of artificial vortex air generator for green electric power, MATEC Web of Conferences, EDP Sciences, 2017, p. 02009.

https://doi.org/10.1051/matecconf/201713102009

[13] H. Al-Kayiem, A. Mustafa, S. Gilani, Vortex field simulation and analysis of a solar updraft power engine, WIT Trans. Ecol. Environ. 2016; 205: 193-202. https://doi.org/10.2495/EQ160181

[14] M. Zhang, X. Luo, T. Li, L. Zhang, X. Meng, K. Kase, S. Wada, CW. Yu, Z. Gu, From dust devil to sustainable swirling wind energy, Scientific reports 2015; 5(1): 1-5. https://doi.org/10.1038/srep08322

[15] N. Ninic, S. Nizetic, Solar power plant with short diffuser, patent WO2009/060245 A 1 (2009).
[16]

Ž. Penga, S. Nižetić, M. Arıcı, Solar plant with short diffuser concept: Further improvement of numerical model by included influence of guide vane topology on shape and stability of gravitational vortex, Journal of cleaner production 2019; 212: 353-361. https://doi.org/10.1016/j.jclepro.2018.12.021

[17] S. Nižetić, Ž. Penga, M. Arıcı, Contribution to the research of an alternative energy concept for carbon free electricity production: Concept of solar power plant with short diffuser, Energy Conversion and Management 2017; 148: 533-553. https://doi.org/10.1016/j.enconman.2017.05.062

[18] A. Mohiuddin, E. Uzgoren, Computational analysis of a solar energy induced vortex generator, Applied Thermal Engineering 2016; 98: 1036-1043. https://doi.org/10.1016/j.applthermaleng.2016.01.005

[19] L. Zuo, N. Qu, L. Ding, P. Dai, Z. Liu, B. Xu, Y. Yuan, A vortex-type solar updraft power-desalination integrated system, Energy Conversion and Management 2020; 222: 113216. https://doi.org/10.1016/j.enconman.2020.113216

[20] S. Nizetic, F. Grubisic-Cabo, M. Bugarin, Experimental Setup for the Analysis of Vortices, Journal of Applied Fluid Mechanics 2015; 8(1).

https://doi.org/10.36884/jafm.8.01.22587

[21] MNA. Saadunand NAC. Sidik, Experimental Study on the Performance of Small Solar Updraft Tower in the ClimateRegion.International Journal OF Automotive AND Mechanical Engineering 2020; 17(4): 8372-8383 https://doi.org/10.15282/ijame.17.4.2020.13.0633

[22] N. Jamaludin, NI. Mohammed, MF. Khamidi, SNA. Wahab, Thermal comfort of residential building in Malaysia at different micro-climates, Procedia-Social and Behavioral Sciences 2015; 170: 613-623.

https://doi.org/10.1016/j.sbspro.2015.01.063

\section{DOI: https://doi.org/10.31875/2410-2199.2021.08.10}

(C) 2021 Tukkee et al.; Zeal Press.

This is an open access article licensed under the terms of the Creative Commons Attribution Non-Commercial License (http://creativecommons.org/licenses/by-nc/3.0/), which permits unrestricted, non-commercial use, distribution and reproduction in any medium, provided the work is properly cited. 